\title{
Detecting and Filtering Immune-Related Adverse Events Signal Based on Text Mining and Observational Health Data Sciences and Informatics Common Data Model: Framework Development Study
}

Yue Yu ${ }^{1}$, PhD; Kathryn Ruddy ${ }^{2}$, MD; Aaron Mansfield ${ }^{2}$, MD; Nansu Zong ${ }^{1}$, PhD; Andrew Wen ${ }^{1}$, MSc; Shintaro Tsuji $1^{1}, \mathrm{PhD}$; Ming Huang ${ }^{1}, \mathrm{PhD}$; Hongfang Liu ${ }^{1}, \mathrm{PhD}$; Nilay Shah ${ }^{1}, \mathrm{PhD}$; Guoqian Jiang ${ }^{1}, \mathrm{MD}, \mathrm{PhD}$

${ }^{1}$ Department of Health Sciences Research, Mayo Clinic, Rochester, MN, United States

${ }^{2}$ Division of Medical Oncology, Department of Oncology, Mayo Clinic, Rochester, MN, United States

\section{Corresponding Author:}

Guoqian Jiang, MD, PhD

Department of Health Sciences Research

Mayo Clinic

200 First St SW

Rochester, MN, 55905

United States

Phone: 15072842511

Email: jiang.guoqian@mayo.edu

\begin{abstract}
Background: Immune checkpoint inhibitors are associated with unique immune-related adverse events (irAEs). As most of the immune checkpoint inhibitors are new to the market, it is important to conduct studies using real-world data sources to investigate their safety profiles.
\end{abstract}

Objective: The aim of the study was to develop a framework for signal detection and filtration of novel irAEs for 6 Food and Drug Administration-approved immune checkpoint inhibitors.

Methods: In our framework, we first used the Food and Drug Administration's Adverse Event Reporting System (FAERS) standardized in an Observational Health Data Sciences and Informatics (OHDSI) common data model (CDM) to collect immune checkpoint inhibitor-related event data and conducted irAE signal detection. OHDSI CDM is a standard-driven data model that focuses on transforming different databases into a common format and standardizing medical terms to a common representation. We then filtered those already known irAEs from drug labels and literature by using a customized text-mining pipeline based on clinical text analysis and knowledge extraction system with Medical Dictionary for Regulatory Activities (MedDRA) as a dictionary. Finally, we classified the irAE detection results into three different categories to discover potentially new irAE signals.

Results: By our text-mining pipeline, 490 irAE terms were identified from drug labels, and 918 terms were identified from the literature. In addition, of the 94 positive signals detected using CDM-based FAERS, 53 signals (56\%) were labeled signals, 10 $(11 \%)$ were unlabeled published signals, and $31(33 \%)$ were potentially new signals.

Conclusions: We demonstrated that our approach is effective for irAE signal detection and filtration. Moreover, our CDM-based framework could facilitate adverse drug events detection and filtration toward the goal of next-generation pharmacovigilance that seamlessly integrates electronic health record data for improved signal detection.

(JMIR Med Inform 2020;8(6):e17353) doi: 10.2196/17353

\section{KEYWORDS}

immunotherapy/adverse effects; drug-related side effects and adverse reactions; pharmacovigilance; adverse drug reaction reporting systems/standards; text mining 


\section{Introduction}

\section{Background}

Immunotherapy activates a patient's immune system for therapeutic benefit against cancer [1]. One type of immunotherapy, immune checkpoint inhibition, has recently been found to be promising for the treatment of certain types of cancer. Immune checkpoint inhibitors can block negative regulators (checkpoints) of T-cell function that exist on both immune and tumor cells. This blockage could enhance antitumor immunity by allowing T cells to kill cancer cells [2]. Notably, the Nobel Prize in Physiology or Medicine in 2018 was awarded to James Allison and Tasuku Honjo for their work on immune checkpoint inhibitors [3]. From 2011 to 2017, the US Food and Drug Administration (FDA) has approved a total of 6 immune checkpoint-directed antibodies for the treatment of specific tumors. By increasing the activity of the immune system, immune checkpoint inhibitors can have inflammatory side effects, which are often termed as immune-related adverse events (irAEs) [4]. The most recognized irAEs include dermatitis, colitis, hepatitis, pancreatitis, pneumonitis, and hypophysitis [5]. IrAEs are mostly of mild to moderate severity, but at times, these can be serious, irreversible, or even fatal. Nevertheless, several studies have indicated that immune checkpoint inhibitors have a better safety profile than many traditional chemotherapies [6-8]. As these immune checkpoint inhibitor agents are new to the market, investigation of their safety profiles in real-world practice is critical [4]. Traditionally, one of the most important ways to detect postmarketing safety profiles of drugs is to conduct pharmacovigilance studies using a spontaneous reporting system (SRS) database [9]. SRS is a system whereby case reports of adverse drug events (ADEs) are voluntarily submitted by health professionals and pharmaceutical companies to the national pharmacovigilance center [10]. Several studies have focused on the irAEs post marketing pharmacovigilance through the analysis of an SRS database such as the US Food and Drug Administration's Adverse Event Reporting System (FAERS) or World Health Organization (WHO)'s VigeBase [11-15].

Although there have been some previous studies that utilized SRS to detect irAEs, it is still essential to investigate new irAE signals to help the research community recognize a comprehensive drug safety profile for these immune checkpoint antibodies. However, it is now also recognized that traditional SRS-based ADE detection methods only focus on detecting statistically significant drug-event pairs from the SRS database, and these methods often face challenges in identifying those new pharmacovigilance signals automatically. Hauben and Aronson [16] proposed a widely used definition of a drug safety surveillance signal. This definition is also issued by the Council for International Organizations of Medical Sciences [17], an international organization established jointly by WHO and the United Nations Educational, Scientific and Cultural Organization, which is famous for establishing guidelines for international pharmacovigilance. According to this definition, a pharmacovigilance signal "represents an association that is new and important, or a new aspect of a known association, and has not been previously investigated and refuted." We can note that a detected drug-event association that is not fully recognized by the previous investigation could be seen as a signal in this definition. These new signals can be considered to be valuable starting points for further investigation and validation. However, for a current large-scale FAERS-based pharmacovigilance study, most of the detected drug-event associations are already recognized by the existing knowledge. Some of these signals have been discovered by clinical trials before approval or by the postmarketing pharmacovigilance study [18]. To filter the known drug-event associations, health care professionals often have to manually review a substantial number of drug safety-related texts, such as drug labels or biomedical literature, to determine whether these novel ADE signals are worthy of further validation [19-21]. It is typically laborious and imprecise to manually review these ADE signals, despite the promising results achieved by existing studies, such as those by Xu et al $[22,23]$ and Yeleswarapu et al [24]. However, these studies have focused on ranking and finding the most significant detection results and did not consider identification of novel ADE signals, which are worth further investigation. Text-mining methods allow for a more efficient way to filter the drug-event associations that are already known by existing knowledge, by extracting known ADEs from drug labels and literature. By automatically filtering out existing signals, this effort can not only discover novel irAE signals detected by the FAERS but may also reduce the labor involved in human intervention.

\section{Objectives}

The objective of this study was to develop a framework for novel signal detection and filtration of irAEs. First, we normalized the FAERS using the Observational Health Data Sciences and Informatics (OHDSI) CDM to improve data standardization and quality to facilitate data collection and analysis. To detect irAEs, we selected all the 6 immune checkpoint inhibitors approved by the FDA before 2018 as our research object. We collected all standardized adverse event data regarding the 6 immune checkpoint inhibitors. Then, the reporting odds ratio (ROR) is utilized to detect the irAEs signal. To filter out those already known irAEs, a customized text-mining pipeline is implemented using clinical text analysis and knowledge extraction system (cTAKES) with MedDRA as a dictionary. Finally, we classified the irAE detection results into three different categories, including potentially new irAE signals.

\section{Methods}

\section{Materials}

\section{Food and Drug Administration's Adverse Event Reporting System}

The FAERS [25] is a database that contains information on adverse event and medication error reports submitted to FDA. FAERS is designed to support postmarketing safety surveillance. All voluntary adverse event reports in FAERS could be submitted by health care professionals (such as physicians, pharmacists, nurses, and others), consumers (such as patients, family members, lawyers, and others), and manufacturers. There are 7 tables in the FAERS database, which includes patient 
demographic table, drug table, adverse reaction table, patient outcome table, report source table, drug therapy table, and indication table. FAERS will update quarterly and can be downloaded from the FDA website. The adverse event name in FAERS is standardized by MedDRA, a rich and highly specific standardized medical terminology. However, the drug name in FAERS is not standard, which may be a drug ingredient name, a brand name, a clinical drug component, or even a spelling error. Some other information such as drug unit and drug dosage are also nonstandard. Therefore, it is important to conduct the data preprocessing to normalize the data in FAERS before the implementation of adverse event signal detection. In this study, we used the FAERS data covering the period from September 2012 to March 2017.

\section{Observational Health Data Sciences and Informatics Common Data Model}

The OHDSI common data model (CDM) [26], also known as Observational Medical Outcomes Partnership CDM, is a data model designed for the systematic analysis of disparate observational databases. OHDSI CDM focuses on transforming different observational databases into a common format (data model) and a common representation (terminologies, vocabularies, coding schemes). As of February 23, 2108, version V5.3 of the CDM was released, containing 37 tables in 6 categories: standardized clinical data, standardized health system data, standardized health economics, standardized metadata, standardized vocabularies, and standardized derived elements. In fact, terminology normalization enabled by standard vocabularies with a focus on systematized nomenclature of medicine-clinical terms (SNOMED CT), logical observation identifiers names and codes, and RxNorm is a strong characteristic of the OHDSI CDM. One of the advantages of using a CDM-based database to conduct a pharmacovigilance study is that we could build a standard query as the same standard terminologies are utilized to represent the medical concepts across the different observational databases. This allows for collaborative pharmacovigilance research across different institutions.

\section{Food and Drug Administration Drug Label}

We searched the DailyMed website to collect the drug labels of 6 FDA-approved immune checkpoint inhibitors [27]. DailyMed, developed and maintained by the National Library of Medicine, is the official provider of FDA drug label information. We downloaded the drug labels of the 6 immune checkpoint inhibitors in January 2018. These drug labels were downloaded in the structured product labeling (SPL) format, which is a document markup standard approved by Health Level Seven (HL7) and adopted by the FDA as a mechanism for exchanging product and facility information. We extracted the text under the section WARNINGS AND PRECAUTIONS and the section ADVERSE REACTIONS from the SPL files of 6 labels as the dataset of drug label text mining.

\section{Immune-Related Adverse Events-Related PubMed Literature}

We retrieved literature from PubMed [28] and built an irAE-related literature text-mining dataset. The query "immune-related [All Fields] AND adverse [All Fields] AND events [All Fields]" (retrieve date: January 2018) was used to retrieve literature from PubMed. A total of 679 irAEs-related literature was obtained. Then, we downloaded the abstract of 679 papers and the full text of 20 review articles as the irAE-related literature text-mining dataset.

\section{Methods}

Using FAERS standardized in the OHDSI CDM, we developed a framework for signal detection and filtration of irAEs, as shown in Figure 1. The framework mainly contains 4 modules as follows (data standardization module, signal detection module, text-mining module, and signal filtration module).

Figure 1. System architecture of our standards-driven framework. ADE: adverse drug events; CDM: common data model; cTAKES: clinical text analysis and knowledge extraction system; FAERS, Food and Drug Administration's adverse event reporting system; IrAEs: immune-related adverse events; MedDRA: medical dictionary for regulatory activities; OHDSI: observational health data sciences and informatics; ROR: reporting odds ratio.

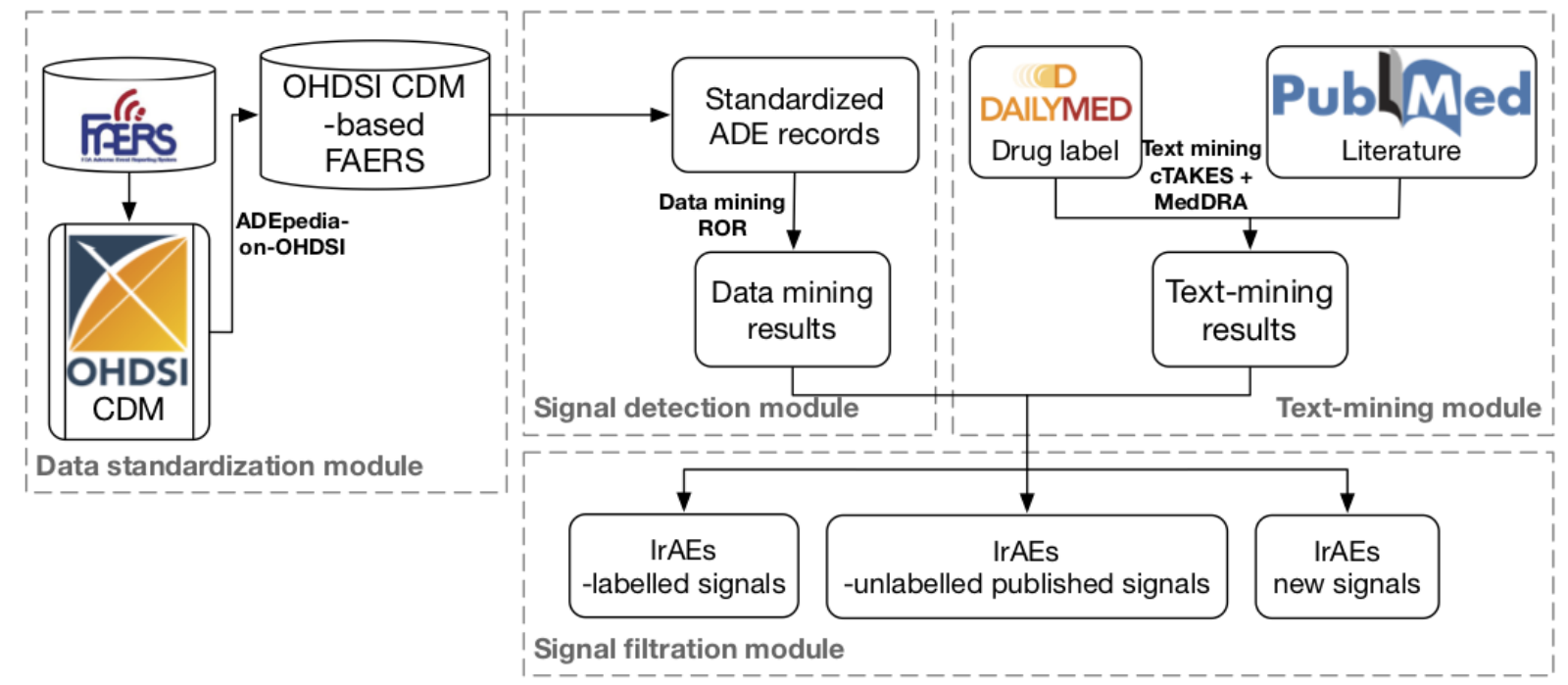




\section{Data Standardization Module}

In FAERS, some data are nonstandard. For example, a drug name in FAERS might be a drug ingredient name, a brand name, a clinical drug component, or even a spelling error. This data standardization problem would cause inconvenience in data collection and integration and introduce bias in data analysis. In this study, we developed a next-generation pharmacovigilance signal detection platform, ADEpedia-on-OHDSI [29], to standardize FAERS and integrate it with electronic health record (EHR) data by OHDSI CDM. Specifically, we used and extended adverse event open learning through universal standardization (AEOLUS) - an integration process developed by Banda et al [30] to develop an extract, transform, and load (ETL) process to transform FAERS data into OHDSI CDM. AEOLUS focuses on building a standard process for FAERS data deduplication and tooling for mapping drug names to RxNorm concepts and outcomes to SNOMED CT concepts.
We further developed an ETL tool to convert FAERS into OHDSI CDM by data structure mapping, medical concept mapping, and data imputation. The 3-step ETL process of the ADEpedia-on-OHDSI platform is shown in Figure 2. (1) Data cleaning and drug name mapping: we used AEOLUS to conduct data deduplication and drug name mapping. (2) Structure mappings between FAERS schema and OHDSI CDM schema: we created structure mappings by choosing appropriate tables or fields between the OHDSI CDM and FAERS. (3) Data ETL implementation: we designed different ETL strategies and then loaded the raw FAERS data into the OHDSI CDM. More details about the ETL process of the ADEpedia-on-OHDSI platform can be found in our published paper [29]. After the ETL process, all the standardized FAERS data were stored in the relational database in the OHDSI CDM format. In this study, we utilized pgAdmin 4 (The pgAdmin Development Team) to operate and maintain our ADEpedia-on-OHDSI platform.

Figure 2. Extract, transform, and load process of converting Food and Drug Administration's Adverse Events Reporting System into Observational Health Data Sciences and Informatics common data model. AEOLUS, adverse event open learning through universal standardization; CDM, common data model; ETL: extract, transform, and load; FAERS, Food and Drug Administration's adverse event reporting system; OHDSI, observational health data sciences and informatics.

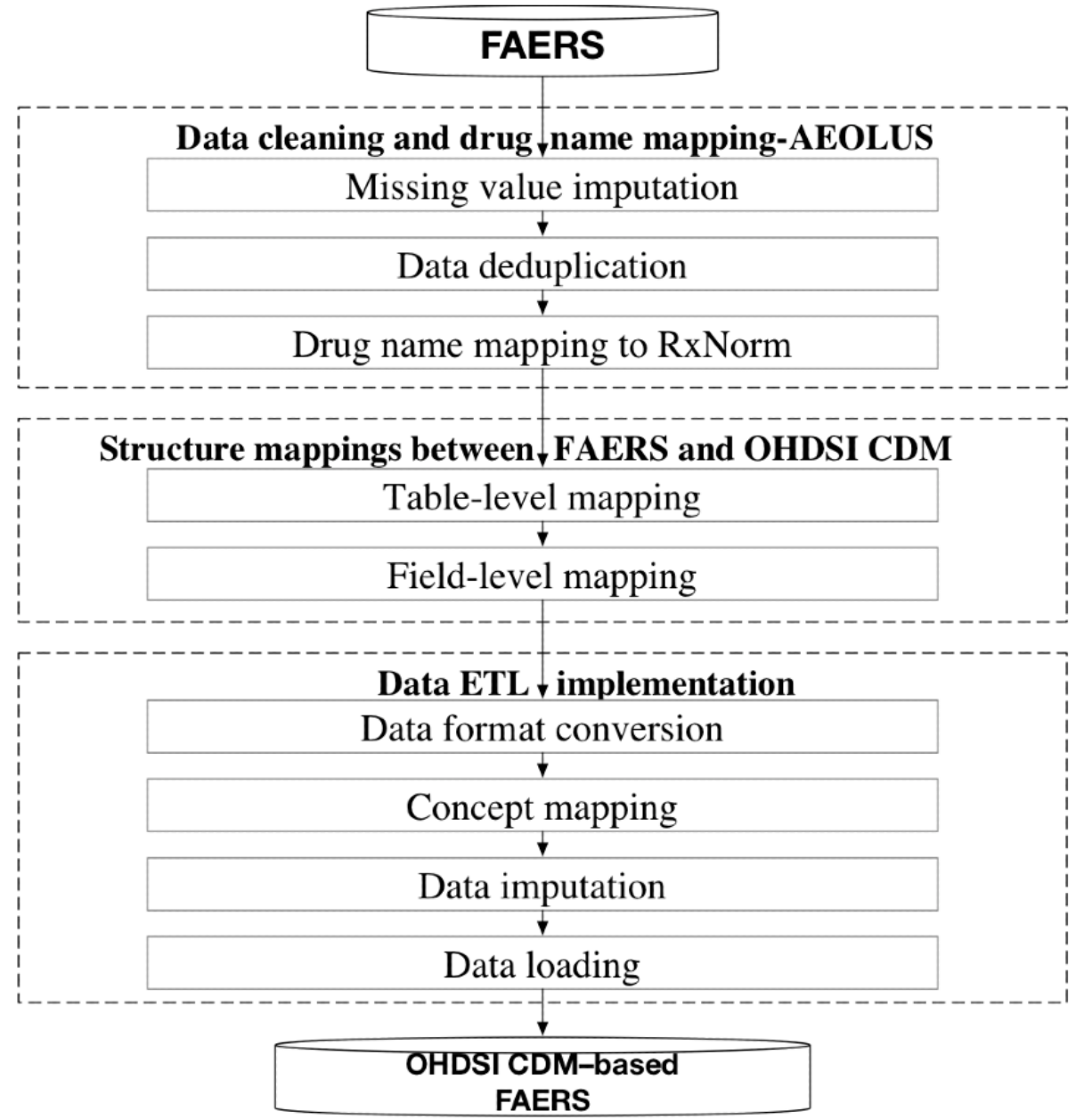

\section{Signal Detection Module}

In this research, we define the adverse drug event (ADE) signal as the significant drug-adverse event associations detected by the detection algorithm using FAERS data. We implemented signal detection algorithms to detect potential irAE signals related to the 6 immune checkpoint inhibitor drugs approved by the FDA (ie, ipilimumab, pembrolizumab, nivolumab, atezolizumab, durvalumab, and avelumab). The active ingredient drug name, brand name, and the corresponding standard concept of those drugs are shown in Table 1. In addition, to facilitate the collection of irAE reports from our CDM-based FAERS, we built a standard query by checking all the synonyms of the 6 standard drug concepts in OHDSI ATHENA standardized 
vocabularies [31]. OHDSI concept_id related to all the ingredient/brand names is used to build SQL queries to retrieve the drug-event reports in our ADEpedia-on-OHDSI platform. The standardized SQL query for the retrieval of irAE reports is described in Multimedia Appendix 1. In addition, to validate our retrieval query, we also implemented a search by using drug ingredient/brand name verbatim texts and compared the results by different retrieval queries.
For ADE signal detection, the ROR [32] was implemented. ROR is one of the most commonly used disproportionality statistical analysis for signal detection in SRSs such as FAERS [33]. Figure 3 illustrates the contingency table and the equation of the ROR. The ROR value and its $95 \%$ CIs were calculated to detect irAE signals. When the case report number was $\geq 3$ and the lower limit of $95 \%$ CI of ROR was $>1$, the signal was considered as a positive irAE signal.

Table 1. The basic information of 6 immune checkpoint inhibitors.

\begin{tabular}{lllll}
\hline $\begin{array}{l}\text { Immune check- } \\
\text { point inhibitor }\end{array}$ & $\begin{array}{l}\text { Brand } \\
\text { name }\end{array}$ & $\begin{array}{l}\text { Food and Drug Administra- } \\
\text { tion-approved year }\end{array}$ & $\begin{array}{l}\text { The Observational Health Data Sciences and } \\
\text { Informatics concept_id (ingredient/brand name) }\end{array}$ & $\begin{array}{l}\text { RxNorm concept unique identifier (ingre- } \\
\text { dient/brand name) }\end{array}$ \\
\hline Ipilimumab & $\begin{array}{l}\text { Yer- } \\
\text { voy }\end{array}$ & 2011 & $40238188 / 40238070$ & $1094833 / 1094837$ \\
Pembrolizumab & $\begin{array}{l}\text { Keytru- } \\
\text { da }\end{array}$ & 2014 & $45775965 / 45775969$ & $1547545 / 1547550$ \\
Nivolumab & $\begin{array}{l}\text { Opdi- } \\
\text { vo }\end{array}$ & 2014 & $45892628 / 45892632$ & $1597876 / 1597881$ \\
Atezolizumab & $\begin{array}{l}\text { Tecen- } \\
\text { triq }\end{array}$ & 2016 & $42629079 / 42629083$ & $1792776 / 1792781$ \\
Imfinzi & 2017 & $1594034 / 1594039$ & $1919503 / 1919508$ \\
Durvalumab & Baven- 2017 & $1593273 / 1593278$ \\
cio & & & $1875534 / 1875543$ \\
\hline
\end{tabular}

Figure 3. The contingency table and equation for the implementation of the reporting odds ratio.

\begin{tabular}{|l|c|c|}
\hline & Reports with target event & Reports without target event \\
\hline $\begin{array}{l}\text { Reports with immune } \\
\text { checkpoint inhibitor }\end{array}$ & $\mathrm{a}$ & $\mathrm{b}$ \\
\hline $\begin{array}{l}\text { Reports without immune } \\
\text { checkpoint inhibitor }\end{array}$ & $\mathrm{c}$ & $\mathrm{d}$ \\
\hline
\end{tabular}

$$
\mathrm{ROR}=\frac{a / b}{c / d}
$$

\section{Text-Mining Module}

We developed a customized text-mining pipeline to identify irAEs from the text of drug labels and irAEs-related literature using cTAKES v4.0. cTAKES is a widely used clinical information extraction tool that can discover clinically named entities and clinical events using a dictionary lookup algorithm [34]. Moreover, we implemented cTAKES using MedDRA as the dictionary to conduct text mining, so that the adverse events extracted could be standardized by MedDRA-preferred terms (PTs). Note that the result of data mining is signal because they have statistical significance, whereas the results extracted by text-mining pipeline are called irAE terms.

For drug label text mining, we collected the drug labels of the 6 FDA-approved immune checkpoint inhibitors from the DailyMed website [27]. These drug labels were downloaded in the SPL format, which is a document markup standard approved by HL7 and adopted by the FDA as a mechanism for exchanging product and facility information. Multimedia Appendix 2 shows the drug label links in DailyMed. Then, we extracted the text under the section WARNINGS AND PRECAUTIONS and the section ADVERSE REACTIONS from the SPL files of 6 labels as the dataset of drug label text mining. In addition, to evaluate the performance of cTAKES in our irAE text-mining pipeline, 2 authors (KR and GJ) manually reviewed the text under those 2 sections of the 6 drug labels and identified the irAE terms out of the drug label text, and they reached consensus via discussions. Both KR and GJ have medical backgrounds, and $\mathrm{KR}$ is a medical oncologist with both clinical and research expertise in treatment toxicities. The irAE terms identified from the manual review were used as a gold standard to assess the baseline performance of our text-mining pipeline, and standard measures (precision, recall, and $F$-measure) were calculated for the performance evaluation.

To identify irAEs from related literature, we searched the PubMed using the query "immune-related[All Fields] AND adverse[All Fields] AND events[All Fields]." A total of 679 irAE-related studies were found, and the abstracts were downloaded for all search results (as of January 2018). We also extracted the text from the full text of 20 review papers from the search results for text mining. The distribution of 
irAE-related literature by year is illustrated in Table 2, showing a trend that the number of studies on irAEs has increased significantly in recent years. We then implemented the text-mining pipeline with MedDRA as a dictionary to extract the irAE terms from both the abstracts and the full review papers of the irAE-related literature.

Table 2. The distribution of literature on immune-related adverse events by year (PubMed retrieve date: January 24, 2018).

\begin{tabular}{ll}
\hline Publication year & Publication number \\
\hline 2006 & 1 \\
2007 & 1 \\
2008 & 5 \\
2009 & 7 \\
2010 & 7 \\
2011 & 11 \\
2012 & 11 \\
2013 & 37 \\
2014 & 47 \\
2015 & 74 \\
2016 & 150 \\
2017 & 260 \\
2018 & 68 \\
\hline
\end{tabular}

\section{Signal Filtration Module}

We reviewed all irAE signals that were identified from the signal detection and classified them into 3 categories: labeled signals (ie, those signals that could be validated by drug labels), unlabeled published signals (ie, signals that could not be found in drug labels, but in published literature), and new signals (ie, signals that could not be found either in drug labels or published literature). Then, 2 oncologists (KR and AM) manually reviewed the new signals category and gave their comments about whether an irAE signal in that category could be seen as a potentially new signal. Note that those oncologists only reviewed the detection results, and they did not have access to any other clinical data to help them ascertain what might be due to the cancer or the treatment.

\section{Results}

\section{Data Standardization Results}

After the ETL process, raw FAERS data were loaded into 8 OHDSI CDM tables. A total of 4,619,362 adverse event case reports were transferred into the OHDSI CDM. Among these patients, 2,577,989 (55.81\%) were female, 1,603,982 (34.72\%) were male, and the sex of 437,391 (9.47\%) was unknown/not specified.

Table 3 shows the total numbers of irAE reports in the raw FAERS and CDM-based FAERS. It should be noted that one patient may receive more than one immune checkpoint inhibitor. We found that more irAE reports were collected after the ETL process. In CDM-based FAERS, a total of 24,595 immune checkpoint inhibitor-related AE reports were collected, compared with 24,500 in the raw FAERS before the ETL process. Of the 6 immune checkpoint inhibitors, nivolumab (Opdivo) had the most AE reports $(\mathrm{n}=12,557$ before ETL and $\mathrm{n}=12,569$ after ETL), followed by ipilimumab (Yervoy; $\mathrm{n}=8264$ before ETL and $n=8268$ after ETL). 
Table 3. Total report numbers of 6 immune checkpoint inhibitors.

\begin{tabular}{llll}
\hline Immune checkpoint inhibitor & $\begin{array}{l}\text { Brand } \\
\text { name }\end{array}$ & $\begin{array}{l}\text { Adverse drug event report } \\
\text { number (before extract, } \\
\text { transform, and load) }\end{array}$ & Adverse drug event report number (after extract, transform, and load) \\
\hline Ipilimumab & Yervoy & 8264 & 8268 \\
Pembrolizumab & Keytruda & 5020 & 5099 \\
Nivolumab & Opdivo & 12,557 & 12,569 \\
Atezolizumab & Tecentriq & 891 & 893 \\
Durvalumab & Imfinzi & 27 & 27 \\
Avelumab & Bavencio & 5 & 5 \\
Total reports & $\mathrm{N} / \mathrm{A}^{\mathrm{a}}$ & 24,500 & 24,595 \\
\hline
\end{tabular}

${ }^{\mathrm{a}} \mathrm{N} / \mathrm{A}$ : not applicable.

\section{Immune-Related Adverse Events Signal Detection Results}

To provide a comprehensive perspective for irAEs, we conducted irAE signal detection at 2 different MedDRA adverse event levels: the system organ class (SOC) level and the PT level. SOC level is the highest level of MedDRA, which contains 27 groupings by etiology (eg, SOC infections and infestations), manifestation site (eg, SOC gastrointestinal disorders), and purpose (eg, SOC surgical and medical procedures). A PT term is a distinct descriptor (single medical concept) that is linked to at least one SOC. Table 4 shows the 7 positive signals detected in the SOC level.

Moreover, 94 positive signals in the PT level were detected in patients who used 1 of the 6 immune checkpoint inhibitor drugs. Among all the positive irAE signals, hypophysitis had the highest ROR value (ROR 5398.8; 95\% CI 3105.1-9386.9), followed by hypopituitarism (ROR 135.1; 95\% CI 106.7-171.1), blood corticotrophin decreased (ROR 59.5; 95\% CI
3105.1-9386.9), adrenal insufficiency (ROR 36.1; 95\% CI 31.5-41.3), and colitis (ROR 32.7; 95\% CI 30.5-35.0), which means these irAEs were possibly suffered most often by the patients who were immune checkpoint inhibitors.

We also classified the irAE signals using the MedDRA SOCs to obtain a high-level understanding of the distribution of the irAE signals (shown in Table 5). Note that 1 PT might be linked to more than 1 SOC, so the total signal number in Table 5 was more than 94. All the signals we detected at the PT level could be classified into 19 SOCs. Moreover, 14 PT-level signals were categorized in Respiratory, thoracic and mediastinal disorders, which is the SOC with the most signals, followed by Gastrointestinal disorders, Cardiac disorders, Infections and infestations, and Nervous system disorders, of which SOCs also had more than 10 PT level signals. In addition, there was at least one PT-level signal in each of the 7 SOCs we previously detected as a positive SOC-level signal, which also validated our detection results at the SOC level. The detailed information of the 94 irAE signals is illustrated in Multimedia Appendix 3.

Table 4. The signal detection results at the system organ class level.

\begin{tabular}{lll}
\hline Medical Dictionary for Regulatory Activities code & System organ class & $\begin{array}{l}\text { Reporting odds ratio } \\
(95 \% \text { CI })\end{array}$ \\
\hline 10014698 & Endocrine disorders & $2.98(2.84-3.12)$ \\
10019805 & Hepatobiliary disorders & $2.53(2.39-2.68)$ \\
10027433 & Metabolism and nutrition disorders & $1.76(1.69-1.83)$ \\
10005329 & Blood and lymphatic system disorders & $1.56(1.48-1.64)$ \\
10029104 & Neoplasms benign, malignant, and unspecified (including & $1.38(1.30-1.46)$ \\
10038738 & cysts and polyps) & $1.27(1.23-1.31)$ \\
10017947 & Respiratory, thoracic, and mediastinal disorders & $1.16(1.12-1.19)$ \\
\hline
\end{tabular}


Table 5. System organ class distribution of preferred term-level signals.

\begin{tabular}{|c|c|}
\hline System organ class & Signal number \\
\hline Respiratory, thoracic, and mediastinal disorders ${ }^{\mathrm{a}}$ & 14 \\
\hline Gastrointestinal disorders $^{\mathrm{a}}$ & 13 \\
\hline Cardiac disorders & 10 \\
\hline Infections and infestations & 10 \\
\hline Nervous system disorders & 10 \\
\hline General disorders and administration site conditions & 9 \\
\hline Investigations & 9 \\
\hline Immune system disorders & 8 \\
\hline Endocrine disorders ${ }^{\mathrm{a}}$ & 5 \\
\hline Hepatobiliary disorders $^{\mathrm{a}}$ & 5 \\
\hline Injury, poisoning, and procedural complications & 5 \\
\hline Metabolism and nutrition disorders ${ }^{\mathrm{a}}$ & 5 \\
\hline Skin and subcutaneous tissue disorders & 5 \\
\hline Blood and lymphatic system disorders ${ }^{\mathrm{a}}$ & 4 \\
\hline Eye disorders & 4 \\
\hline Musculoskeletal and connective tissue disorders & 4 \\
\hline Vascular disorders & 4 \\
\hline Renal and urinary disorders & 3 \\
\hline $\begin{array}{l}\text { Neoplasms benign, malignant, and unspecified (including cysts and } \\
\text { polyps) }\end{array}$ & 1 \\
\hline
\end{tabular}

${ }^{\mathrm{a}}$ Represents the system organ class that was detected as a positive signal in the system organ class level.

\section{Text-Mining Results}

As mentioned previously, we utilized cTAKES with MedDRA as a dictionary to identify the irAE terms from the drug label of 6 immune checkpoint inhibitors. A total of 421 and 918 irAE terms were found by text mining of drug labels and irAEs-related literature, respectively.

Regarding drug label text mining, we found that most of the irAE terms identified by cTAKES were in the PT level of MedDRA. However, some of the irAE terms were defined as lowest-level terms (LLTs) in MedDRA. An LLT is a synonym, lexical variant, quasi-synonym, subelement, or an identical to its related PT and could be linked to only one PT. To unify the irAE terms to standard concepts at the same level, we mapped all the LLTs into PTs based on the recommendations of MedDRA and FDA. As a result, 490 irAE terms were extracted from the texts of all the 6 drug labels, comprising 474 PTs, 15 SOC, and 1 high-level term (HLT, a superordinate descriptor for the PTs linked to it). More details of the irAE terms identified from drug labels by our text-mining pipeline are provided in Multimedia Appendix 4.

For the text-mining evaluation, as mentioned in the Methods section, the irAE terms manually identified by 2 experts (KR and GJ) from drug labels were seen as the gold standard. Then, irAE terms extracted by the text-mining pipeline were compared with the gold standard to acquire the text-mining performance. As the text-mining pipeline, we also linked all the LLT-level irAE terms to the PT level. Using the expert-based manual review process, we identified a total of 421 distinct irAE terms from drug labels of the 6 immune checkpoint inhibitors, comprising 401 PTs, 10 SOCs, 1 HLT, and 9 terms that could not be mapped with MedDRA concepts. Multimedia Appendix 5 provides the details of the manually identified irAE terms. Table 6 shows the distribution of the irAE terms in different drug labels and the performance of our text-mining pipeline. As illustrated in the table, the overall precision, recall, and $F$-measure of our text-mining pipeline are $79.39 \%, 92.40 \%$, and $85.40 \%$, respectively, which indicates that our pipeline could provide satisfactory text-mining results and achieved the requirement of our irAE identification task.

For irAE-related literature text mining, by using our text-mining pipeline, a total of 918 unique irAE terms (in PT or higher level) were identified from 679 irAE-related abstracts and 20 irAE-related review papers, in which $306(33.33 \%)$ terms were covered by the irAE terms that were extracted in drug labels, and the remaining $612(66.67 \%)$ terms were not covered by the labeled irAE terms. This indicates that some unlabeled terms can be identified from our text-mining pipeline. Multimedia Appendix 6 provides the results of irAE-related literature text mining. 
Table 6. Performance of text-mining pipeline for the identification of immune-related adverse events from drug labels of 6 immune checkpoint inhibitors.

\begin{tabular}{|c|c|c|c|c|c|c|c|c|}
\hline $\begin{array}{l}\text { Immune check- } \\
\text { point inhibitor }\end{array}$ & $\begin{array}{l}\text { Manually } \\
\text { identified } \\
\text { immune-re- } \\
\text { lated adverse } \\
\text { events terms }\end{array}$ & $\begin{array}{l}\text { Clinical Text } \\
\text { Analysis and } \\
\text { Knowledge Ex- } \\
\text { traction Sys- } \\
\text { tem-identified } \\
\text { immune-related } \\
\text { adverse events } \\
\text { terms }\end{array}$ & True positive & False positive & False negative & $\begin{array}{l}\text { Precision } \\
\text { (TP/[TP+FP]), } \\
\%\end{array}$ & $\begin{array}{l}\text { Recall } \\
(\mathrm{TP} / \mathrm{TP}+\mathrm{FN}]) \\
\%\end{array}$ & $\begin{array}{l}F \text {-measure } \\
(2 \mathrm{PR} /[\mathrm{P}+\mathrm{R}]) \\
\%\end{array}$ \\
\hline Ipilimumab & 122 & 138 & 103 & 35 & 19 & 74.6 & 84.4 & 79.2 \\
\hline Pembrolizumab & 192 & 228 & 179 & 49 & 13 & 78.5 & 93.2 & 85.2 \\
\hline Nivolumab & 215 & 262 & 202 & 60 & 13 & 77.1 & 93.9 & 84.7 \\
\hline Atezolizumab & 142 & 157 & 129 & 28 & 13 & 82.2 & 90.9 & 86.3 \\
\hline Durvalumab & 179 & 183 & 156 & 27 & 23 & 85.3 & 87.2 & 86.2 \\
\hline Avelumab & 146 & 176 & 130 & 46 & 16 & 73.9 & 89.0 & 80.8 \\
\hline Total & 421 & 490 & 389 & 101 & 32 & 79.4 & 92.4 & 85.4 \\
\hline
\end{tabular}

\section{Signal Filtration Results}

To filter the irAE signals we detected, we compared all 94 irAE signals with the text-mining results and then classified all the signals into 3 categories as per our definition in the Methods section. Figure 4 shows the overlap of the irAEs terms identified in 3 different mining tasks. In total, 1135 unique irAE terms were identified by CDM-based FAERS data mining, drug label manual review, and irAE-related literature text mining. Out of 94 positive signals in the PT level detected using CDM-based FAERS, 53 signals (56\%) were the labeled signals we identified from drug labels, 10 signals $(11 \%)$ were the unlabeled published signals identified from the literature, and 31 signals (33\%) were potentially new signals that were not covered by drug labels and literature (as shown in Table 7). Multimedia Appendix 7 demonstrates the details of labeled signals, unlabeled published signals, and new signals. For a further manual review, 2 oncologists separately marked 15 and 8 signals that were possibly new, after reviewing a total of 31 irAE signals in the new signal category. The kappa coefficient value of the review is 0.48 , which showed a moderate agreement between the 2 oncologists [35]. Moreover, 7 irAE signals were identified as potentially new signals by both the oncologists (as shown in Table 7).

Figure 4. Venn diagram illustrating the immune-related adverse events terms detected from different sources. CDM, common data model; FAERS, Food and Drug Administration's adverse event reporting system.

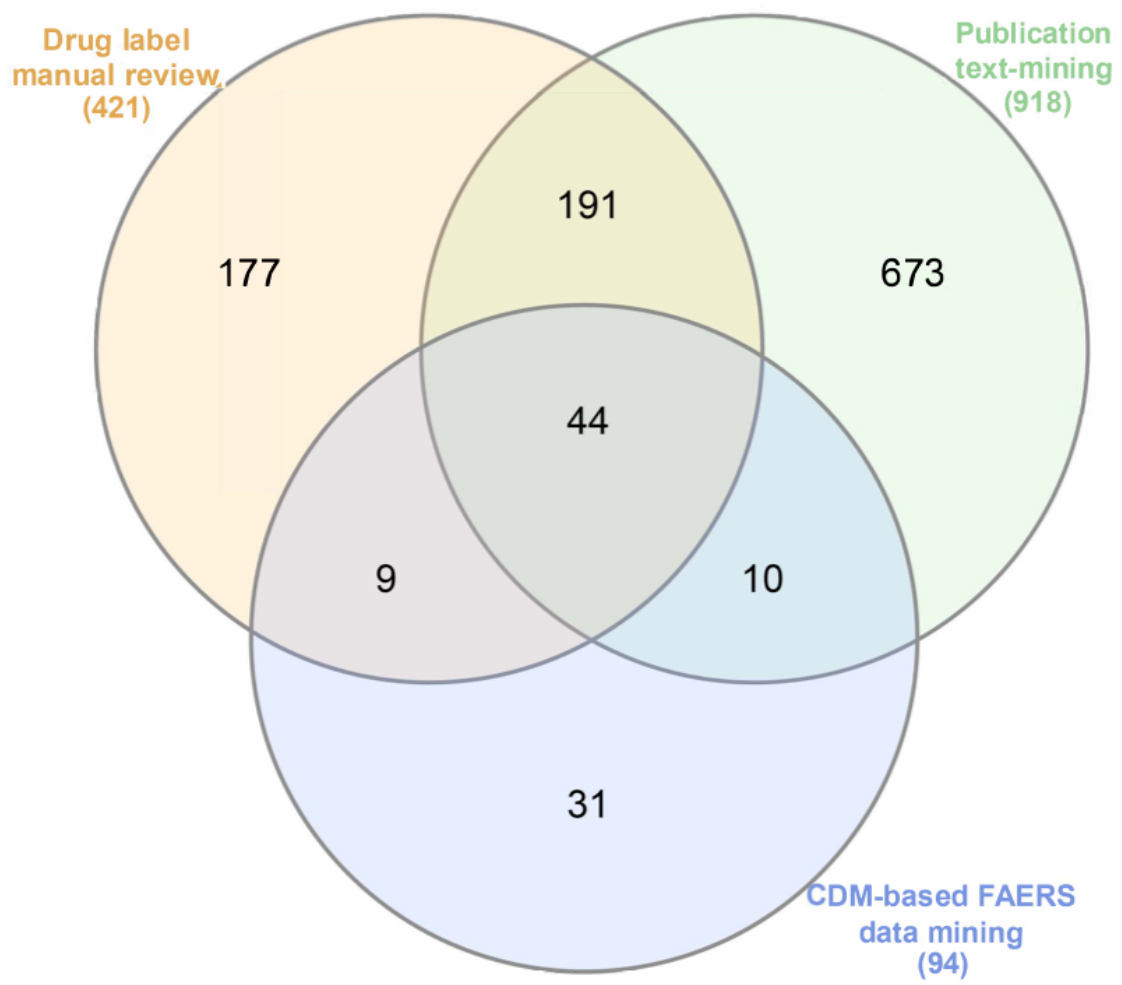


Table 7. A list of 31 potentially new signals not identified in drug labels or literature (ranked by reporting odds ratio).

\begin{tabular}{|c|c|c|c|}
\hline $\begin{array}{l}\text { Medical Dictionary for Regu- } \\
\text { latory Activities code }\end{array}$ & Preferred term & System organ class & Reporting odds ratio $(95 \% \mathrm{CI})$ \\
\hline 10005452 & Blood corticotrophin decreased & Investigations & $59.49(34.44-102.74)$ \\
\hline 10053481 & Bronchopleural fistula $^{\mathrm{a}}$ & $\begin{array}{l}\text { Respiratory, thoracic, and mediastinal } \\
\text { disorders }\end{array}$ & $19.51(6.96-54.67)$ \\
\hline 10006437 & Bronchial fistula $^{\mathrm{a}}$ & $\begin{array}{l}\text { Respiratory, thoracic, and mediastinal } \\
\text { disorders }\end{array}$ & $19.01(6.79-53.20)$ \\
\hline 10042569 & Superior vena cava syndrome & $\begin{array}{l}\text { Vascular disorders/ neoplasms benign, } \\
\text { malignant, and unspecified (including } \\
\text { cysts and polyps) }\end{array}$ & $10.62(5.78-19.51)$ \\
\hline 10061457 & Facial nerve disorder $^{\mathrm{a}}$ & Nervous system disorders & $9.51(3.48-25.97)$ \\
\hline 10044291 & Tracheal obstruction $^{\mathrm{a}}$ & $\begin{array}{l}\text { Respiratory, thoracic, and mediastinal } \\
\text { disorders/ injury, poisoning, and procedu- } \\
\text { ral complications }\end{array}$ & $7.83(2.47-24.87)$ \\
\hline 10058838 & Enterocolitis infectious & $\begin{array}{l}\text { Gastrointestinal disorders/infections and } \\
\text { infestations }\end{array}$ & $7.64(3.77-15.51)$ \\
\hline 10065764 & Mucosal infection & $\begin{array}{l}\text { General disorders and administration site } \\
\text { conditions/infections and infestations }\end{array}$ & $7.13(2.25-22.59)$ \\
\hline 10013832 & Duodenal perforation & Gastrointestinal disorders & $6.50(2.88-14.68)$ \\
\hline 10006440 & Bronchial obstruction $^{\mathrm{a}}$ & $\begin{array}{l}\text { Respiratory, thoracic, and mediastinal } \\
\text { disorders }\end{array}$ & $6.34(3.13-12.82)$ \\
\hline 10061145 & Eyelid function disorder ${ }^{\mathrm{a}}$ & Eye disorders & $5.73(1.82-18.09)$ \\
\hline 10007196 & Capillary leak syndrome $^{\mathrm{a}}$ & $\begin{array}{l}\text { General disorders and administration site } \\
\text { conditions/vascular disorders }\end{array}$ & $5.62(2.78-11.35)$ \\
\hline 10010276 & Conduction disorder & Cardiac disorders & $4.92(2.19-11.07)$ \\
\hline 10036774 & Proctitis & Gastrointestinal disorders & $4.90(2.82-8.50)$ \\
\hline 10021305 & Ileal perforation & Gastrointestinal disorders & $4.09(1.30-12.84)$ \\
\hline 10009995 & Colonic fistula & Gastrointestinal disorders & $3.81(1.21-11.95)$ \\
\hline 10064774 & Infusion site extravasation & $\begin{array}{l}\text { Injury, poisoning, and procedural compli- } \\
\text { cations/general disorders and administra- } \\
\text { tion site conditions }\end{array}$ & $3.51(2.30-5.36)$ \\
\hline 10051341 & Bile duct stenosis & Hepatobiliary disorders & $3.45(1.42-8.35)$ \\
\hline 10042241 & Stridor & $\begin{array}{l}\text { Respiratory, thoracic, and mediastinal } \\
\text { disorders }\end{array}$ & $3.15(1.41-7.06)$ \\
\hline 10035623 & Pleuritic pain & $\begin{array}{l}\text { Respiratory, thoracic, and mediastinal } \\
\text { disorders }\end{array}$ & $3.12(1.67-5.82)$ \\
\hline 10025256 & Lymphocyte count decreased & Investigations & $2.97(2.29-3.85)$ \\
\hline 10063057 & Cystitis noninfective & Renal and urinary disorders & $2.83(1.05-7.60)$ \\
\hline 10005630 & $\begin{array}{l}\text { Blood lactate dehydrogenase in- } \\
\text { creased }\end{array}$ & Investigations & $2.81(2.10-3.76)$ \\
\hline 10041549 & Spinal cord compression & Nervous system disorders & $2.81(1.66-4.76)$ \\
\hline 10008612 & Cholecystitis & Hepatobiliary disorders & $2.59(1.87-3.58)$ \\
\hline 10041103 & Small intestinal perforation & Gastrointestinal disorders & $2.46(1.02-5.94)$ \\
\hline 10003662 & Atrial flutter & Cardiac disorders & $2.45(1.50-4.02)$ \\
\hline 10036206 & Portal vein thrombosis ${ }^{\mathrm{a}}$ & Vascular disorders/hepatobiliary disorders & $2.43(1.30-4.53)$ \\
\hline 10029164 & Nephrotic syndrome & Renal and urinary disorders & $2.37(1.42-3.94)$ \\
\hline 10003673 & Atrioventricular block complete & Cardiac disorders & $1.85(1.09-3.13)$ \\
\hline
\end{tabular}




\begin{tabular}{llll}
\hline $\begin{array}{l}\text { Medical Dictionary for Regu- } \\
\text { latory Activities code }\end{array}$ & Preferred term & System organ class & Reporting odds ratio (95\% CI) \\
\hline 10003504 & Aspiration & $\begin{array}{l}\text { Respiratory, thoracic, and mediastinal } \\
\text { disorders }\end{array}$ & $1.60(1.02-2.51)$ \\
\hline
\end{tabular}

${ }^{\mathrm{a}}$ Identified as potentially new signals by both oncologist reviewers.

\section{Discussion}

\section{Principal Findings}

To the best of our knowledge, this is the first comprehensive, novel signal detection and filtration study of irAEs utilizing multiple drug safety data sources. We proposed a framework to detect the irAE signals from a standardized FAERS database and utilized a text-mining pipeline with drug labels and existing literature to discover potentially new irAE signals. Our framework could facilitate ADE detection and filtration toward the goal of next-generation pharmacovigilance. This could decrease the labor consumption in new irAE signal selection and provide stronger hypotheses for further experimental validation. In the future, the results of this work will be potentially combined with the EHR data to leverage the real-world discovery of treatment toxicities.

We utilized standard OHDSI CDM to represent the FAERS data (ie, the ADEpedia-on-OHDSI platform) and created standard queries for signal detection, which provides a solid data infrastructure to make the queries portable and signal detection results reproducible. More importantly, through the comparison of data collection between the raw FAERS and CDM-based FAERS, we found that the OHDSI CDM could improve the precision of the data collection. For example, for the drug pembrolizumab, we collected 5099 reports from the CDM-based FAERS, 79 reports more than those we collected from the raw FAERS. To illustrate the reason for the difference in data collection using the OHDSI CDM-based FAERS, we manually checked the data we collected from the raw FAERS and CDM-based FAERS. For example, we discovered that when we utilized the standard OHDSI concept id as a query to retrieve CDM-based FAERS, we could collect more reports regarding the drug name "MK-3475," which was the original name of pembrolizumab in its early development, in addition to those reports we retrieved when we used the drug ingredient name "Pembrolizumab" and brand name "Keytruda." This meant that we improved the true positive rate and precision for data collection. Moreover, we could save time for collecting data through a standard query. For example, for pembrolizumab, it took 9.4 seconds to pull all data from CDM-based FAERS with our standard query, in contrast to approximately 70 seconds for the raw FAERS data collection through a fuzzy search query with the drug/brand name terms.

We also leveraged text-mining technology to process unstructured drug safety data. We implemented our text-mining pipeline on the drug label and irAE literature with MedDRA as a dictionary to identify the irAE terms. In addition, to evaluate the performance of our text-mining pipeline, the irAEs in drug labels were manually reviewed and extracted as a gold standard. As a result, the overall precision, recall, and $F$-measure of all 6 drug labels were $79.39 \%, 92.40 \%$, and $85.40 \%$, respectively.
These results indicate that although there were some false positive terms (about 20\%) found in our text-mining results, most irAE terms $(92.40 \%)$ in the text could be extracted correctly by our pipeline. Moreover, we checked the underlying reasons behind the false positive terms. We found that most of these terms are related to the laboratory test name, such as Alanine aminotransferase, Blood alkaline phosphatase, and so on. Actually, for laboratory tests, the appropriate terms matched with irAEs should be the specific abnormal test result terms, such as Alanine aminotransferase increased and Blood alkaline phosphatase increased, which were also found in both gold standard and text-mining results. Given this analysis, we plan to improve the precision of our text-mining pipeline using a rule-based approach in a future update.

\section{Limitations and Future Work}

Our framework provides an automatic process to detect novel irAE signals that are more valuable for implementing further experimental validation. It also profoundly saves the experts' time in reviewing drug labels and the literature to filter the known ADEs. In total, we detected 94 irAE signals from FAERS. After the filtering, 31 irAEs were classified into the new irAE signals category. In addition, 7 out of 31 signals in the new signal category were identified as potentially new irAE signals by both the oncologists, which indicated that some of the new signals detected by these algorithms might be false positive. According to the oncologists' review, some of the signals were marked as not new. We consider that one of the main reasons for the false positive signal was that sometimes the description of irAEs by the MedDRA PTs was not so accurate. For example, some detected new signals might be a hyponym of a known irAE, that is, they are more specific than a general irAE. For example, Conduction disorder and Atrioventricular block complete were detected as new irAE signals by our pipeline. However, the oncologist reviewers judged that these are not new because they are types of arrhythmias, which belong to cardiotoxicity and are known to be associated with the immune checkpoint blockade [36]. Moreover, 2 of the potentially new signals, bronchopleural fistula/bronchial fistula and tracheal obstruction/bronchial obstruction, are almost the same medical concept. Thus, there is a need to develop a harmonized terminology to report and describe irAEs to interpret safety data more accurately in monitoring missions. One of our previous studies discussed the possibility of leveraging the Common Terminology Criteria for Adverse Events (CTCAE) for irAE standardization. We found that the CTCAE needs an extension to meet the irAE standardization task [37]. Similarly, other studies have also demonstrated how to build a terminology to standardize irAEs $[38,39]$. In future work, we will improve the text-mining process to facilitate the development of ADE terminology. First, to create a harmonized irAE terminology and make it more suitable for detecting irAE signals from other data sources such as EHR, 
we will extend the text-mining dictionary to SNOMED CT. Second, we will further improve the performance and automation of our text-mining pipeline to make the terminology easier to update and maintain. Third, we will further evaluate the text-mining pipeline to investigate its feasibility of developing specific terminology sets for other ADE categories.

For those irAE signals in the new signal category, some of them were marked as possibly new by 2 oncologists because these adverse events may be induced by or associated with cancer, the complication of surgery or radiation, other drugs administered in the cancer treatment regimen, or drug- drug interactions. For example, Bronchopleural fistula, bronchial obstruction, and bronchial fistula all can occur due to a pulmonary cancer or as a complication of pulmonary surgery or radiation [40]. However, FAERS does not provide information such as timeline details about the drug administration/diagnosis/event, which is an obstacle to confirming whether a signal is caused by the treatment or other conditions. Therefore, expert reviews from oncologists are important for our detection pipeline to control false positive signal results. Moreover, as mentioned in the Methods section, oncologists also need more clinical data to further validate the relationship between these irAE signals and immunotherapy drugs. Longitudinal observational databases such as EHRs have increasingly been used for further evaluation of adverse event signals. Compared with FAERS data, EHRs not only contain information about patients who suffer ADEs but also provide a more complete medical history of the patients, including treatments, conditions, and potential risk factors. Accordingly, EHRs could be an additional data source for irAE signal detection [41]. We are actively working on integrating EHR data with our ADEpedia-on-OHDSI platform, which can scale to support more advanced signal detection [42]. Our standard-driven platform integrates FAERS data and EHR data together by using the same data standards that could facilitate pharmacovigilance research based on real-world data. Our platform can not only improve data quality but can also facilitate the data collection for comprehensive ADE detection or cross-validation. In the future, we will try to conduct more comprehensive ADE detection studies based on real-world data to overcome the false positive issue. Furthermore, we will also consider utilizing semantic web technology to develop more ADE mining methods.

\section{Conclusions}

In this study, we developed and evaluated a novel standards-based framework for signal detection and filtration of irAEs using both the OHDSI CDM and text-mining technologies. We demonstrated that our approach is effective for novel irAE signal detection and filtration; meanwhile, the CDM-based platform provides an infrastructure that would enable the seamless integration of EHR data for improving signal detection in the future.

\section{Acknowledgments}

This research was funded in part by National Institutes of Health U01 HG009450. The authors would like to thank Na Hong and Liwei Wang for their previous work in FAERS standardization.

\section{Conflicts of Interest}

AM reports research support from Novartis and Verily; remuneration to his institution for participation on advisory boards for AbbVie, Astra Zeneca, BMS, and Genentech; and travel support from Roche and is a nonremunerated director of the Mesothelioma Applied Research Foundation.

\section{Multimedia Appendix 1}

The standardized SQL query for the irAE record retrieving.

[DOCX File, 13 KB-Multimedia Appendix 1]

\section{Multimedia Appendix 2}

The drug label links of six FDA-approved mAb drugs in DailyMed. [DOCX File, 13 KB-Multimedia Appendix 2]

\section{Multimedia Appendix 3}

The detail information of 94 irAE signals at PT level.

[XLS File (Microsoft Excel File), 44 KB-Multimedia Appendix 3]

\section{Multimedia Appendix 4}

The irAE terms identified form drug labels by the text-mining pipeline.

[XLSX File (Microsoft Excel File), $61 \mathrm{~KB}-$ Multimedia Appendix 4]

\section{Multimedia Appendix 5}

The detail of the manually identified irAE terms from the drug labels. 
[XLSX File (Microsoft Excel File), 54 KB-Multimedia Appendix 5]

\section{Multimedia Appendix 6}

The irAE terms identified form irAE-related literature by the text-mining pipeline.

[XLSX File (Microsoft Excel File), 41 KB-Multimedia Appendix 6]

\section{Multimedia Appendix 7}

The detail of labeled irAE signals, unlabeled published irAE signals, and new irAE signals.

[XLSX File (Microsoft Excel File), 28 KB-Multimedia Appendix 7]

\section{References}

1. Mellman I, Coukos G, Dranoff G. Cancer immunotherapy comes of age. Nature 2011 Dec 21;480(7378):480-489 [FREE Full text] [doi: 10.1038/nature10673] [Medline: 22193102]

2. Friedman CF, Proverbs-Singh TA, Postow MA. Treatment of the immune-related adverse effects of immune checkpoint inhibitors: a review. JAMA Oncol 2016 Oct 1;2(10):1346-1353. [doi: 10.1001/jamaoncol.2016.1051] [Medline: 27367787]

3. Kaiser J, Couzin-Frankel J. Cancer immunotherapy sweeps nobel for medicine. Science 2018 Oct 5;362(6410):13. [doi: 10.1126/science.362.6410.13] [Medline: 30287641]

4. Postow MA, Sidlow R, Hellmann MD. Immune-related adverse events associated with immune checkpoint blockade. N Engl J Med 2018 Jan 11;378(2):158-168. [doi: 10.1056/NEJMra1703481] [Medline: 29320654]

5. Sharma P, Allison JP. Immune checkpoint targeting in cancer therapy: toward combination strategies with curative potential. Cell 2015 Apr 9;161(2):205-214 [FREE Full text] [doi: 10.1016/j.cell.2015.03.030] [Medline: 25860605]

6. Postow MA, Callahan MK, Wolchok JD. Immune checkpoint blockade in cancer therapy. J Clin Oncol 2015 Jun 10;33(17):1974-1982 [FREE Full text] [doi: 10.1200/JCO.2014.59.4358] [Medline: 25605845]

7. Borghaei H, Paz-Ares L, Horn L, Spigel DR, Steins M, Ready NE, et al. Nivolumab versus docetaxel in advanced nonsquamous non-small-cell lung cancer. N Engl J Med 2015 Oct 22;373(17):1627-1639 [FREE Full text] [doi: 10.1056/NEJMoa1507643] [Medline: 26412456]

8. Reck M, Rodríguez-Abreu D, Robinson AG, Hui R, Csőszi T, Fülöp A, KEYNOTE-024 Investigators. Pembrolizumab versus chemotherapy for PD-L1-positive non-small-cell lung cancer. N Engl J Med 2016 Nov 10;375(19):1823-1833. [doi: 10.1056/NEJMoa1606774] [Medline: 27718847]

9. Harpaz R, DuMouchel W, Shah NH, Madigan D, Ryan P, Friedman C. Novel data-mining methodologies for adverse drug event discovery and analysis. Clin Pharmacol Ther 2012 Jun;91(6):1010-1021 [FREE Full text] [doi: 10.1038/clpt.2012.50] [Medline: 22549283]

10. World Health Organization. The Safety of Medicines in Public Health Programmes - Pharmacovigilance: An Essential Tool. Geneva, Switzerland: WHO Publications; 2006.

11. Garcia CR, Cox JN, Villano JL. Myasthenia gravis and Guillain-Barré syndrome adverse events with immune checkpoint inhibitors. J Clin Oncol 2018 Feb 10;36(5_suppl):37. [doi: 10.1200/jco.2018.36.5_suppl.37]

12. Elias R, Rider J, Tan X, Rahma OE. Single agent and combination checkpoint inhibitors therapy: a post marketing safety analysis. J Clin Oncol 2018 Feb 10;36(5_suppl):125. [doi: 10.1200/jco.2018.36.5 suppl.125]

13. Moslehi JJ, Salem J, Sosman JA, Lebrun-Vignes B, Johnson DB. Increased reporting of fatal immune checkpoint inhibitor-associated myocarditis. Lancet 2018 Mar 10;391(10124):933 [FREE Full text] [doi: 10.1016/S0140-6736(18)30533-6] [Medline: 29536852]

14. Al-Kindi SG, Oliveira GH. Reporting of immune checkpoint inhibitor-associated myocarditis. Lancet 2018 Aug 4;392(10145):382-383. [doi: 10.1016/S0140-6736(18)31542-3] [Medline: 30102167]

15. Oshima Y, Tanimoto T, Yuji K, Tojo A. EGFR-TKI-associated interstitial pneumonitis in nivolumab-treated patients with non-small cell lung cancer. JAMA Oncol 2018 Aug 1;4(8):1112-1115 [FREE Full text] [doi: 10.1001/jamaoncol.2017.4526] [Medline: 29327061]

16. Hauben M, Aronson JK. Defining 'signal' and its subtypes in pharmacovigilance based on a systematic review of previous definitions. Drug Saf 2009;32(2):99-110. [doi: 10.2165/00002018-200932020-00003] [Medline: 19236117]

17. Council for International Organizations of Medical Sciences. Practical Aspects of Signal Detection in Pharmacovigilance: Report of CIOMS Working Group VIII. Geneva, Switzerland: WHO Publications; 2010.

18. Coloma PM, Trifirò G, Patadia V, Sturkenboom M. Postmarketing safety surveillance: where does signal detection using electronic healthcare records fit into the big picture? Drug Saf 2013 Mar;36(3):183-197. [doi: 10.1007/s40264-013-0018-x] [Medline: 23377696]

19. Huang J, Zhang X, Tong J, Du J, Duan R, Yang L, et al. Comparing drug safety of hepatitis C therapies using post-market data. BMC Med Inform Decis Mak 2019 Aug 8;19(Suppl 4):147 [FREE Full text] [doi: 10.1186/s12911-019-0860-6] [Medline: $\underline{31391106]}$ 
20. Rahman MM, Alatawi Y, Cheng N, Qian J, Peissig PL, Berg RL, et al. Methodological considerations for comparison of brand versus generic versus authorized generic adverse event reports in the US food and drug administration adverse event reporting system (FAERS). Clin Drug Investig 2017 Dec;37(12):1143-1152 [FREE Full text] [doi: 10.1007/s40261-017-0574-4] [Medline: 28933038]

21. Singh P, Nayernama A, Jones SC, Kordestani LA, Fedenko K, Prowell T, et al. Fatal neutropenic enterocolitis associated with docetaxel use: a review of cases reported to the United States food and drug administration adverse event reporting system. J Oncol Pharm Pract 2019 Oct 8:- epub ahead of print. [doi: 10.1177/1078155219879494] [Medline: 31594460]

22. $\mathrm{Xu} \mathrm{R}$, Wang Q. Large-scale combining signals from both biomedical literature and the FDA adverse event reporting system (FAERS) to improve post-marketing drug safety signal detection. BMC Bioinformatics 2014 Jan 15;15:17 [FREE Full text] [doi: 10.1186/1471-2105-15-17] [Medline: 24428898]

23. Xu R, Wang Q. Automatic signal extraction, prioritizing and filtering approaches in detecting post-marketing cardiovascular events associated with targeted cancer drugs from the FDA adverse event reporting system (FAERS). J Biomed Inform 2014 Mar;47:171-177 [FREE Full text] [doi: 10.1016/j.jbi.2013.10.008] [Medline: 24177320]

24. Yeleswarapu S, Rao A, Joseph T, Saipradeep VG, Srinivasan R. A pipeline to extract drug-adverse event pairs from multiple data sources. BMC Med Inform Decis Mak 2014 Mar 24;14:13 [FREE Full text] [doi: 10.1186/1472-6947-14-13] [Medline: 24559132]

25. openFDA. FDA Adverse Event Reporting System URL: https://open.fda.gov/data/faers/ [accessed 2020-05-06]

26. OHDSI: Observational Health Data Sciences and Informatics. OHDSI Common Data Model URL: https://ohdsi.org/ [accessed 2020-05-06]

27. DailyMed. DailyMed: NIH URL: https://dailymed.nlm.nih.gov/dailymed/ [accessed 2020-05-06]

28. PubMed-NCBI. PubMed URL: https://www.ncbi.nlm.nih.gov/pubmed/ [accessed 2020-05-06]

29. Yu Y, Ruddy KJ, Hong N, Tsuji S, Wen A, Shah ND, et al. ADEpedia-on-OHDSI: a next generation pharmacovigilance signal detection platform using the OHDSI common data model. J Biomed Inform 2019 Mar;91:103119 [FREE Full text] [doi: 10.1016/j.jbi.2019.103119] [Medline: 30738946]

30. Banda JM, Evans L, Vanguri RS, Tatonetti NP, Ryan PB, Shah NH. A curated and standardized adverse drug event resource to accelerate drug safety research. Sci Data 2016 May 10;3:160026 [FREE Full text] [doi: 10.1038/sdata.2016.26] [Medline: $\underline{27193236]}$

31. Athena: OHDSI. License Agreement URL: http://athena.ohdsi.org [accessed 2020-05-06]

32. van Puijenbroek EP, Bate A, Leufkens HG, Lindquist M, Orre R, Egberts AC. A comparison of measures of disproportionality for signal detection in spontaneous reporting systems for adverse drug reactions. Pharmacoepidemiol Drug Saf 2002;11(1):3-10. [doi: 10.1002/pds.668] [Medline: 11998548]

33. Bate A, Evans SJ. Quantitative signal detection using spontaneous ADR reporting. Pharmacoepidemiol Drug Saf 2009 Jun;18(6):427-436. [doi: 10.1002/pds.1742] [Medline: 19358225]

34. Savova GK, Masanz JJ, Ogren PV, Zheng J, Sohn S, Kipper-Schuler KC, et al. Mayo clinical text analysis and knowledge extraction system (cTAKES): architecture, component evaluation and applications. J Am Med Inform Assoc 2010;17(5):507-513 [FREE Full text] [doi: 10.1136/jamia.2009.001560] [Medline: 20819853]

35. Landis JR, Koch GG. The measurement of observer agreement for categorical data. Biometrics 1977 Mar;33(1):159-174. [Medline: $\underline{843571]}$

36. Hassel JC, Heinzerling L, Aberle J, Bähr O, Eigentler TK, Grimm M, et al. Combined immune checkpoint blockade (anti-PD-1/anti-CTLA-4): evaluation and management of adverse drug reactions. Cancer Treat Rev 2017 Jun;57:36-49. [doi: 10.1016/j.ctrv.2017.05.003] [Medline: 28550712]

37. Yu Y, Ruddy KJ, Tsuji S, Hong N, Liu H, Shah N, et al. Coverage evaluation of CTCAE for capturing the immune-related adverse events leveraging text mining technologies. AMIA Jt Summits Transl Sci Proc 2019;2019:771-778 [FREE Full text] [Medline: 31259034$]$

38. Wang Q, Xu R. Immunotherapy-related adverse events (irAEs): extraction from FDA drug labels and comparative analysis. JAMIA Open 2019 Apr;2(1):173-178 [FREE Full text] [doi: 10.1093/jamiaopen/ooy045] [Medline: 30976759]

39. Zini EM, Lanzola G, Quaglini S, Cornet R. Standardization of immunotherapy adverse events in patient information leaflets and development of an interface terminology for outpatients' monitoring. J Biomed Inform 2018 Jan;77:133-144 [FREE Full text] [doi: 10.1016/j.jbi.2017.12.009] [Medline: 29269275]

40. Lois M, Noppen M. Bronchopleural fistulas: an overview of the problem with special focus on endoscopic management. Chest 2005 Dec;128(6):3955-3965. [doi: 10.1378/chest.128.6.3955] [Medline: 16354867]

41. Harpaz R, Vilar S, Dumouchel W, Salmasian H, Haerian K, Shah NH, et al. Combing signals from spontaneous reports and electronic health records for detection of adverse drug reactions. J Am Med Inform Assoc 2013 May 1;20(3):413-419 [FREE Full text] [doi: 10.1136/amiajnl-2012-000930] [Medline: 23118093]

42. Yu Y, Ruddy K, Wen A, Zong N, Shintaro T, Chen J, et al. Integrating electronic health record data into the ADEpedia-on-OHDSI platform for improved signal detection: a case study of immune-related adverse events. AMIA 2020 Informatics Summit 2019:710-719 (forthcoming) [FREE Full text] 


\title{
Abbreviations
}

ADE: adverse drug event

AEOLUS: adverse event open learning through universal standardization

CDM: common data model

cTAKES: clinical text analysis and knowledge extraction system

CTCAE: common Terminology Criteria for Adverse Events

EHR: electronic health record

ETL: extract, transform, and load

FAERS: Food and Drug Administration's Adverse Event Reporting System

FDA: Food and Drug Administration

HL7: Health Level Seven

HLT: high-level term

irAEs: immune-related adverse events

LLTs: lowest-level terms

MedDRA: Medical Dictionary for Regulatory Activities

OHDSI: Observational Health Data Sciences and Informatics

PT: preferred term

ROR: reporting odds ratio

SNOMED CT: systematized nomenclature of medicine-clinical terms

SOC: system organ class

SPL: structured product labeling

SQL: Structured Query Language

SRS: spontaneous reporting system

WHO: World Health Organization

\author{
Edited by G Eysenbach; submitted 09.12.19; peer-reviewed by R Richesson, $S$ Hume, K Tingay, CH Lin, B Kijsanayotin; comments \\ to author 27.03.20; revised version received 13.04.20; accepted 15.04.20; published 12.06.20 \\ Please cite as: \\ $Y$ u Y, Ruddy K, Mansfield A, Zong N, Wen A, Tsuji S, Huang M, Liu H, Shah N, Jiang G \\ Detecting and Filtering Immune-Related Adverse Events Signal Based on Text Mining and Observational Health Data Sciences and \\ Informatics Common Data Model: Framework Development Study \\ JMIR Med Inform 2020;8(6):e17353 \\ URL: http://medinform.jmir.org/2020/6/e17353/ \\ doi: $10.2196 / 17353$ \\ PMID: $\underline{32530430}$
}

(C) Yue Yu, Kathryn Ruddy, Aaron Mansfield, Nansu Zong, Andrew Wen, Shintaro Tsuji, Ming Huang, Hongfang Liu, Nilay Shah, Guoqian Jiang. Originally published in JMIR Medical Informatics (http://medinform.jmir.org), 12.06.2020. This is an open-access article distributed under the terms of the Creative Commons Attribution License (https://creativecommons.org/licenses/by/4.0/), which permits unrestricted use, distribution, and reproduction in any medium, provided the original work, first published in JMIR Medical Informatics, is properly cited. The complete bibliographic information, a link to the original publication on http://medinform.jmir.org/, as well as this copyright and license information must be included. 